\title{
Estimation of the order of an auto-regressive model
}

\author{
N SUDARSHAN RAO* and P S MOHARIR** \\ *Department of Electronics \& Communication Engineering, Osmania \\ University, Hyderabad 500007, India. \\ **National Geophysical Research Institute, Uppal Road, Hyderabad \\ 500007 , India.
}

MS received 21 April 1994; revised 24 October 1994

\begin{abstract}
There are no definitive solutions available for the order estimation of the auto-regressive process. First, the performance of the three criteria, namely FPE, AIC and MDL is illustrated. Next, it is indicated that there are possibilities of their performance being improved. The algorithm tri proposed here utilizes three minimum values of any of the conventional loss functions in the FPE, AIC and MDL methods. It also uses three statistics derived from these three minimum values. The estimated order is a rounded weighted average of these six statistics. The algorithm is found to do better in a qualified sense of yielding peakier distribution of the estimated orders when tested for 1000 synthetic models of orders 3, 5 and 7 each. The conclusion drawn is that there are open possibilities of improving upon the conventional order estimators for auto-regressive processes. This means that till axiologically sounder estimators are available one should be ready to use heuristic estimators proposed here.
\end{abstract}

Keywords. Algorithm tri; auto-regressive process; order estimation; plural criteria; order statistics.

\section{Introduction}

Consider a time series $x(n), n=0,1, \cdots, N-1$ of finite length $N$. If

$$
x(n)=\sum_{i=1}^{r} \alpha_{r, i} x(n-i)+u(n)
$$

where $u(n)$ is an uncorrelated innovations process, then $x(n)$ is said to be an autoregressive time-series (ARTS) of order $r$ (Box \& Jenkins 1976). Coefficients $\alpha_{r, i}, i=1,2, \cdots, r$ are called auto-regressive or linear prediction coefficients (LPCs). As the true order $r$ would be unknown and would have to be estimated, and as it determines the number of LPCs to be estimated, it is useful to make $r$ an explicit part of 
the notation for LPCs as done above. It also permits the possibility of referring to $r$ LPCs under different assumed orders $r$, pending the estimation of $r$. The innovations process $u(n)$ is assumed to be zero-mean, white and Gaussian.

There are many methods to estimate the order $r$ of ARTS. Three famous ones are final prediction error (FPE) (Akaike 1969, 1970), asymptotic information criterion (AIC) (Akaike 1973, 1974) and minimum description length (MDL)(Rissanen 1983). At times the ' $A$ ' in AIC is taken to be Akaike's rather than asymptotic to give the acronym an eponymic advantage. The basic structure of the three methods is similar. They use a loss function which is minimized. The three loss functions are:

$$
\begin{aligned}
\Lambda_{\mathrm{FPE}}(N, r) & =\hat{\sigma}^{2}(N+r+1) /(N-r-1) . \\
\Lambda_{\mathrm{AIC}}(N, r) & =\ln \hat{\sigma}^{2}+2 r / N \\
\Lambda_{\mathrm{MDL}}(N, r) & =N \ln \hat{\sigma}^{2}+r \ln N .
\end{aligned}
$$

Each loss function depends on $\hat{\sigma}^{2}$ which is the estimated variance of the linear prediction error. This is expected to monotonically decrease as the assumed or fitted order $r$ is increased. Then there is a modifier dependent on $r$, the assumed order and $N$, the length of the timeseries available. The purpose of the modifier is to arrange for a minimum in the loss function, possibly at the correct order. The modifier could be multiplicative as in the case of FPE or additive as in the case of AIC and MDL and a monotone transformation could be used on the variance of the prediction error before a modifier is applied as in the latter two cases.

Most users assume that the problem of estimating the order of the auto-regressive process is solved satisfactorily and use one of the standard methods available for the purpose. One of the aims of this paper is to suggest that the problem above is still an open one. Thus, initially the performance of the FPE, AIC and MDL methods is assessed $a b$ initio. This has not been done adequately in the literature. For example, one study (Shibata 1976) reports the frequency distribution of the estimated order by the AIC method on the basis of 500 realizations when the correct order is unity. For $N=50$ and $\alpha_{1,1}= \pm 0.8$ the order is estimated as unity about $74 \%$ times. When $\alpha_{1,1}$ is weaker the percentage of correct order estimation is even lower. Another more recent study (Broersen \& Wensink 1993) used 10,000 realizations of ARTS of order two and $N=25$ to obtain the frequency distribution of the estimated order by 10 different methods. The percentage of correct order estimation ranged between 35.6 and 74.4. There are many applications wherein the ARTS model of higher orders would be necessary (Makhoul 1975; Kay \& Marple 1981). Therefore, true orders 3,5 and 7 are used here to generate 1000 realizations each of ARTS and for $n=50$, the frequency distribution of the estimated orders has been studied. The inference drawn is that the three conventional methods do not perform very satisfactorily, making their axiological elegance only of restricted practical utility.

The problem posed here is whether the performance of the conventional order estimators can be improved. It is a real problem, particularly in the context that it is not even widely known that the performance of the conventional order estimators is as bad as is brought out here, for values of $r$ between 3 and 7 .

The approach here is heuristic and is substantiated by extensive numerical simulation as better axiology was not available for more than two decades. To keep the computational efforts within manageable limits, the effects of sample size $N$ have not been studied. $N$ is chosen to be 50 which is larger of the values used in the two earlier 
studies (Shibata 1976; Broersen \& Wensink 1993) referred to above. The number of realizations of ARTS used to base the inference on is between the values used in the earlier two studies. The number of methods investigated here also lies in between. Taking these three considerations together, it may be said that the computational determinants of effort here form roughly the geometrical mean of the two earlier studies, given that three as against just one, and that too larger values of the correct order have been studied. Further, the computational effort put in is of the same order as for the latter of the two studies.

Motivation for the present work came through watching the loss functions of the three methods of ARTS order estimation. Unlike the formal expectation from equations (2)-(4), the loss functions do not show a sharp single minimum. Their shapes are at times very irregular and depend on the parameters (LPCs) of the ARTS realization. Therefore, it was believed that more information could be extracted from the loss functions to improve upon the conventional order estimators. The work reported establishes such a hope without claiming that the results here cannot be improved upon in a variety of ways.

\section{Assessment of the conventional estimators}

Thousand ARTS models of orders 3,5 and 7 each have been generated. The basic idea was to make them representative of all the possibilities that could arise. This obviously needs proper definition. ARTS models are amenable to at least three different characterizations. They are in terms of their auto-correlation coefficients, linear prediction coefficients and reflection coefficients. The three characterizations are interconvertible. But it does not mean that they are equivalent for all purposes. For example, it has been shown that when ARTS models are to be ordered on the basis of their proximity to a prescribed model, the results may be different when the three characterizations above are used (Moharir 1993). This obviously means that the characterization to be relied upon must be chosen a priori. The characterization chosen here is in terms of the reflection coefficients. The reflection coefficient $(\mathrm{RC})$

$$
k_{j}=\alpha_{j . j}
$$

is the last LPC when an ARTS model of order $j$ is imposed on the time series. There are some good reasons why the $\mathrm{RCs}$ have been chosen for a proper characterization of the ARTS models. When the Levinson-Durbin algorithm (LDA) (Kay \& Marple 1981; Moharir 1985; Alexander 1986) is used for estimating the LPCs, reflection coefficients turn out to be important intermediate results (Makhoul 1975). But even more pertinently, the prediction on the basis of the ARTS model can be arranged entirely in terms of the RCs (Makhoul 1975; Kay \& Marple 1981) with some advantages. Further, the Schur-Cohn stability condition (Proakis 1989) for the ARTS is that

$$
\left|k_{j}\right|<1 \text {. }
$$

Thus, the appropriate $\mathrm{RC}$ space is a hypercube. The stability conditions are more involved in terms of the other two characterizations of ARTS. Filling a hypercubical region uniformly with uncorrelated random numbers is possible and easy but it has been suggested (Hoel 1966) that for regions with other boundaries it may not even be possible in principle. Therefore, the definition of a set of models representative of all 
Table 1. The assessment of the performance of the three methods, FPE, AIC and MDL, for the estimation of the order of the ARTS models.

The numbers of models which gave the estimated order are tabulated against the latter. Thousand synthetic models of true ARTS orders 3, 5 and 7 each were synthesized. See text for the non-numerical entries in the column 'estimated order'. Entries against them summarize the properties of the distribution of the estimated orders.

\begin{tabular}{|c|c|c|c|c|c|c|c|c|c|}
\hline \multirow{2}{*}{$\begin{array}{l}\text { Estimated } \\
\text { order }\end{array}$} & \multicolumn{3}{|c|}{ Correct order $=3$} & \multicolumn{3}{|c|}{ Correct order $=5$} & \multicolumn{3}{|c|}{ Correct order $=7$} \\
\hline & FPE & AIC & MDL & FPE & $\mathrm{AIC}$ & MDL & FPE & AIC & MDL \\
\hline 0 & 38 & 38 & 66 & 12 & 12 & 31 & 4 & 4 & 21 \\
\hline 1 & 73 & 73 & 94 & 28 & 28 & 49 & 13 & 12 & 44 \\
\hline 2 & 186 & 184 & 224 & 45 & 43 & 74 & 31 & 31 & 54 \\
\hline 3 & 607 & 609 & 582 & 132 & 132 & 157 & 61 & 61 & 93 \\
\hline 4 & 67 & 67 & 29 & 169 & 168 & 203 & 71 & 71 & 101 \\
\hline 5 & 24 & 23 & 5 & 465 & 465 & 415 & 108 & 106 & 133 \\
\hline 6 & 3 & 4 & & 80 & 82 & 37 & 197 & 195 & 192 \\
\hline 7 & 3 & 2 & & 58 & 59 & 31 & 340 & 340 & 273 \\
\hline 8 & & & & 8 & 8 & 2 & 107 & 110 & 76 \\
\hline 9 & & & & 1 & 1 & 1 & 56 & 58 & 11 \\
\hline 10 & & & & 1 & 1 & & 19 & 9 & 2 \\
\hline 11 & & & & 1 & 1 & & & 1 & \\
\hline 12 & - & - & - & - & - & - & -- & - & 2 \\
\hline$C$ & 607 & 609 & 582 & 465 & 465 & 415 & 340 & 340 & 273 \\
\hline$L$ & 297 & 295 & 384 & 386 & 383 & 514 & 485 & 480 & 638 \\
\hline$H$ & 97 & 96 & 34 & 149 & 152 & 71 & 183 & 180 & 89 \\
\hline$N$ & 860 & 860 & 835 & 714 & 715 & 655 & 644 & 645 & 541 \\
\hline$N_{0}^{s}$ & 793 & 793 & 806 & 634 & 633 & 618 & 537 & 535 & 465 \\
\hline
\end{tabular}

possibilities was taken to mean a set in which RCs are chosen to spread uniformly and randomly over every axis of the hypercube.

For each of the 3000 ARTS models chosen, LDA was used to evaluate the RCs. A time-series sample of length $N=50$ was used for this purpose. Then predictions were performed under various assumed orders and were compared against the extension of 50 values of the same ARTS to calculate $\hat{\sigma}^{2}$ of (2)-(4). The FPE, AIC and MDL estimates of ARTS order were obtained by minimizing the respective loss functions. The results are shown in table 1 .

In the column for the estimated order in table 1, there are five non-numerical entries at the end. $C$ refers to the correct order. Order 3 is correctly estimated less than $61 \%$ times by the FPE and AIC methods and about $58 \%$ times by the MDL method. For orders 5 and 7 the percentage of correct order estimation is even lower for all the methods. The difference between the performance of the MDL method and the other two also is seen to grow as the correct order increases. For the correct order of 7 , the percentage of correct order estimation is about $34 \%$ for FPE and AIC methods and just about $27 \%$ for the MDL method. $L$ stands for the 'orders lower than the correct one' and $H$ for the 'orders higher than the correct one'. As the correct order increases, for all the three methods the percentage of underestimation or overestimation of the order increases, underestimation being more probable than the overestimation. The MDL method underestimates more and overestimates less than the other two. $N_{s}$ stands for the 'symmetric neighbourhood of the correct order' comprising the 
correct order and immediately adjacent orders, one on each side. When the order is incorrectly estimated, an underestimate may be preferred to an overestimate by the same amount because erroneous as both the estimates are, the former subsequently requires less effort or cost to predict. Further, simpler models have more philosophical appeal for those who adopt Occam's razor (Anderson 1963; Bernal 1969; Constable 1987; Moharir 1991). In this spirit $N_{o}$ stands for the 'Occamic neighbourhood of the correct order' and includes the correct order and the next lower order. On the criteria of the estimated order being in the neighbourhood of the correct order, the three methods do much better than on the criterion of the correct order estimation. Major part of the improvement comes by going to the Occamic neighbourhood from the correct order, the further improvement obtained by enlarging the neighbourhood symmetrically being relatively smaller. The first improvement is larger for the MDL method than for the other two and the second one is relatively small. For the correct order of 3 , the MDL method even does marginally better than the other two on the criterion of $N_{o}$. For all the methods studied, the distributions of the estimated order is asymmetrical with respect to the correct order.

Information summarized in table 1 and some preliminary numerical experiments suggested that the conventional order estimators may possibly be improved upon. One possible way of obtaining the improvement and the attendant difficulties are discussed in the next section.

\section{Algorithm tri}

The loss functions used by the FPE, AIC and MDL methods are motivated by the usefulness of deriving a measure which takes the least value for the correct order. But the above results indicate that it does not happen frequently enough. Further, it is believed to be a common experience with those who observe the loss functions plotted rather than the algorithm reporting the estimated order on their basis that these loss functions are not necessarily uni-minimal. Therefore, it is surmised that there is more information in the loss functions about the order of ARTS than is contained in their lowest minima. The question then was whether it could be extracted. The results presented below should be regarded rather as one such possibility than the final answer.

Table 2. One possibility in which three opinions about the ARTS order can be consensually corrected. The actual correction strategy used is a modification of this.

\begin{tabular}{lcll}
\hline $\begin{array}{l}\text { Ranking of the } \\
\text { three opinions }\end{array}$ & $\begin{array}{l}\text { Possible corrected values } \\
\text { of the three opinions }\end{array}$ \\
\hline$r_{1}<r_{2}<r_{3}$ & $r_{1}$ & $r_{2}-1$ & $r_{3}-2$ \\
$r_{1}<r_{3}<r_{2}$ & $r_{1}$ & $r_{3}-1$ & $r_{2}-2$ \\
$r_{2}<r_{1}<r_{3}$ & $r_{2}+1$ & $r_{1}$ & $r_{3}-1$ \\
$r_{2}<r_{3}<r_{1}$ & $r_{2}+2$ & $r_{3}+1$ & $r_{1}$ \\
$r_{3}<r_{1}<r_{2}$ & $r_{3}+1$ & $r_{1}$ & $r_{2}-1$ \\
$r_{3}<r_{2}<r_{1}$ & $r_{3}+2$ & $r_{2}+1$ & $r_{1}$ \\
\hline
\end{tabular}


From the loss function graphs three order values were chosen which gave the three lowest values of the loss function. Let these orders viewed as three opinions be called $r_{1}$, $r_{2}$ and $r_{3}$. Then $r_{1}$ would be the conventional order estimate. These opinions can be ordered in six different ways as shown on the left of table 2 . If the opinions are to be corrected, as they refer to the same correct order, one possible way of correcting, giving maximum credence to the first opinion, is shown on the right of table 2 . The corrections applied are the least possible, given that the three opinions are different. This can be expressed in a single formula as follows:

$$
P_{j}=r_{j}-\left[R\left(r_{j}\right)-R\left(r_{1}\right)\right], \quad j=1,2,3,
$$

where $R(\cdot)$ is the rank when the three opinions are ranked in the ascending order and $P_{j}$ is the corrected value of the opinion $r_{j}$. Next, it is possible to modify $(7)$ by making the correction depend on the spread of the three opinions. That is, if $r_{\max }$ and $r_{\min }$ are the maximum and minimum values of the order among the three opinions one could choose

$$
P_{j}=r_{j}-\beta_{j}\left(r_{\max }-r_{\min }\right)\left[R\left(r_{j}\right)-R\left(r_{1}\right)\right],
$$

where $\beta_{j}$ is called the degree of correction of $r_{j}$. Further if $P_{j}$ turns out to be negative, it could rather be set to zero. The values of $\beta_{2}$ and $\beta_{3}$ were chosen as $0 \cdot 2$. Corrections applied amount to editing the opinions about the quantity to be estimated for achieving robustness and are in the spirit of Winsorization, trimming and jack kniving (Barnett \& Lewis 1978; Diaconis \& Efron 1983; Moharir 1988).

In addition to the three corrected opinions, three statistics are derived from the three opinions. They are:

$$
\begin{aligned}
& P_{4}=r_{\text {mid }}, \\
& P_{5}=r_{\text {max }}-r_{\text {min }}, \\
& P_{6}=\left(r_{1} r_{2} r_{3}\right)^{1 / 3},
\end{aligned}
$$

where $r_{\text {mid }}$ is the middle value among $r_{1}, r_{2}$ and $r_{3}$. The averages of these three statistics are presumed to represent the central tendency, spread and third cumulant of the ARTS order distribution. Therefore, use of these three statistics is in the nature of stochastic feedback up to the third order moments or cumulants. The rounded value of the weighted average of $P_{j}$ 's, $j=1,2, \cdots, 6$ is taken to be the estimate $r_{\text {est }}$ of the ARTS order. That is,

$$
r_{\text {est }}=\sum_{j=1}^{6} w_{j} P_{j} / \sum_{j=1}^{6} w_{j},
$$

where for convenience the weight factors $w_{j}$ are taken to be nonnegative multiples of 0.25 . It can be readily seen that care has been taken to ensure that the quantities averaged are dimensionally compatible. In fact, the particular measures of central tendency, spread and skewness have been specifically chosen to satisfy this requirement.

It was found that the weight factors cannot be chosen uniquely to achieve that, for a given true order, the order estimates most frequently match the correct order. It was also found that a choice of weight factors which achieves this for a particular true ARTS order is not necessarily likely to achieve it simultaneously for other true orders. Therefore, the numerical experiment was performed as follows. For the true order three, a large number of combinations of weight factors which gave largest or near-largest overall 
Table 3. Summary of the performance of tri-modification for ARTS order estimation, operating on the three conventional methods, viz. FPE, AIC and MDL. For the nonnumerical code for estimated order, see text.

Weight factors (a): $[0 \cdot 25,0 \cdot 75,0 \cdot 25,4 \cdot 25,2,1]$

(b): $[0 \cdot 25,0.5,0 \cdot 5,4 \cdot 25,2,1]$

(c): $[0,0.75,0.5,4,2,1]$

(d): $[0 \cdot 5,0.5,0.5,4,2,1]$

Symbols for methods: tri-modified FPE $=$ TFPE, etc.

\begin{tabular}{llllll}
\hline $\begin{array}{l}\text { Estimated } \\
\text { order code }\end{array}$ & TFPE TAIC TMDL TFPE TAIC TMDL
\end{tabular}

(1) Correct order $=3$

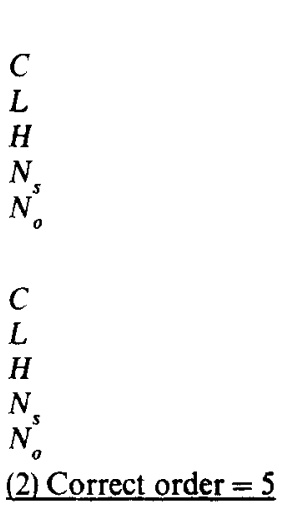

\begin{tabular}{rrr} 
& \multicolumn{2}{c}{ Weight factors $a$} \\
\cline { 2 - 2 } 750 & 749 & 750 \\
161 & 161 & 233 \\
89 & 90 & 17 \\
945 & 944 & 917 \\
868 & 867 & 902 \\
\multicolumn{3}{c}{ Weight factors $c$} \\
750 & 749 & 750 \\
161 & 161 & 233 \\
89 & 90 & 17 \\
945 & 944 & 917 \\
868 & 867 & 902
\end{tabular}

\begin{tabular}{crr} 
& \multicolumn{3}{c}{ Weight factors $b$} & \\
\cline { 2 - 3 } 722 & 721 & 730 \\
161 & 161 & 233 \\
117 & 118 & 37 \\
945 & 945 & 917 \\
840 & 839 & 882 \\
\multicolumn{3}{c}{ Weight factors $d$} \\
722 & 721 & 730 \\
161 & 161 & 233 \\
117 & 118 & 37 \\
945 & 944 & 919 \\
840 & 839 & 884
\end{tabular}

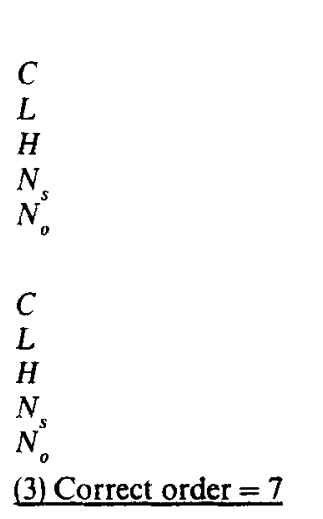

\begin{tabular}{rrr}
\multicolumn{3}{c}{ Weight factors $a$} \\
\cline { 2 - 3 } 403 & 402 & 327 \\
502 & 499 & 615 \\
95 & 99 & 23 \\
767 & 768 & 695 \\
703 & 702 & 679 \\
\multicolumn{3}{c}{ Weight factors $c$} \\
\cline { 2 - 2 } 408 & 407 & 323 \\
497 & 494 & 654 \\
95 & 99 & 23 \\
765 & 768 & 694 \\
703 & 702 & 678
\end{tabular}

\begin{tabular}{rrr}
\multicolumn{3}{c}{ Weight factors $\boldsymbol{b}$} \\
\cline { 2 - 3 } 408 & 407 & 333 \\
497 & 494 & 643 \\
95 & 99 & 24 \\
780 & 782 & 707 \\
718 & 716 & 690 \\
\multicolumn{3}{c}{ Weight factors d } \\
\cline { 2 - 2 } 406 & 405 & 332 \\
499 & 496 & 644 \\
95 & 99 & 24 \\
777 & 779 & 703 \\
715 & 713 & 686
\end{tabular}

\begin{tabular}{|c|c|c|c|c|c|c|}
\hline \multirow[b]{2}{*}{ C } & \multicolumn{3}{|c|}{ Weight factors $a$} & \multicolumn{3}{|c|}{ Weight factors $b$} \\
\hline & 358 & 366 & 211 & 368 & 372 & 212 \\
\hline$L$ & 592 & 589 & 780 & 592 & 579 & 779 \\
\hline$H$ & 50 & 55 & 9 & 40 & 49 & 9 \\
\hline$N$ & 654 & 654 & 489 & 654 & 656 & 488 \\
\hline \multirow[t]{2}{*}{$N_{o}^{s}$} & 612 & 612 & 480 & 622 & 618 & 479 \\
\hline & \multicolumn{3}{|c|}{ Weight factors $c$} & \multicolumn{3}{|c|}{ Weight factors $d$} \\
\hline C & 369 & 373 & 219 & 368 & 374 & 214 \\
\hline$L$ & 592 & 579 & 780 & 592 & 579 & 779 \\
\hline$H$ & 39 & 48 & 9 & 40 & 47 & 7 \\
\hline$N$ & 652 & 654 & 488 & 652 & 654 & 488 \\
\hline$N_{0}^{s}$ & 621 & 617 & 479 & 620 & 620 & 481 \\
\hline
\end{tabular}


percentage of correct order estimation on the basis of the FPE, AIC and MDL loss functions of (2)-(4) were found. This was done on the basis of only one-fifth of the 1000 ARTS models of order 3 to save computational effort. Some hopeful combinations among them were tested for simultaneous good performance for true order 7 and were selected on that basis. Again the test was restricted to only one-seventh of the 1000 ARTS models generated. The results for the true order 5 were then taken as they came.

The numerical comparison is offered in table 3 . The comparison is made for four different choices of weight factors. In one of them, one of the weights is zero. That means only five determinants of order are used. More particularly, the one that is not used is the conventional order estimate, but it does not seem to matter much! This is a very direct suggestion that the loss functions of equations (2)-(4) have more information than or parallel modes of information to the one utilised by the conventional order estimation methods.

What is provided in table 3 is basically the summary in terms of the non-numerical symbols regarding estimated order, introduced in the context of table 1. Comparing the relevant part of table 3 with that of table 1, it can be seen that for the correct ARTS order of 3 , tri algorithm with all the four weight factors yields results better than the conventional algorithm on the criterion $C$ though weight factors (b) and (d) do not do as well as (a) and (c). For the correct ARTS order 5, no weight factor yields tri results superior to the conventional methods on the criterion $C$. For the correct ARTS order 7 , all the weight factors yield tri results better than the FPE and AIC methods. For correct order 7, the tri modification does better on the criterion $N_{o}$ but not on $N_{s}^{\prime}$ for the MDL method. This too is understandable because, of the three conventional methods, MDL has the strongest tendency to underestimate the order. On the criteria $N_{s}$ or $N_{o}$ for the correct ARTS orders 5, all the weight factors yield tri results better than those of the conventional methods. Thus, taking $C$ as the first criterion and $N_{s}$ and/or $N_{o}$ as a supplementary criterion of considerable importance, it is possible to uniformly improve the performance of the conventional order estimators at least for the range of correct ARTS order considered. This uniformly better performance may not be the best at individual values of the correct order but that is just as well because, if the correct order is known, there is no order estimation problem; therefore order estimation algorithms should work over a range of true orders expected.

It should be pointed out that the results reported here are not the best possible because they are not based on proper optimization but only on incomplete search. Also the search for improvement has not been individualized for either the conventional criteria of FPE, AIC and MDL or for criteria $C, N_{s}$ or $N_{o}$. Therefore, the results only hint at the possible inadmissibility of the conventional methods, a method being defined as inadmissible (Judge \& Bock 1978) if it can be uniformly improved upon without any claims about the optimality of what is proposed. The tri algorithm also separates the order estimation problem from that of the estimation of ARTS coefficients. Once the former is solved, the latter also can be reapproached using unconventional techniques (Judge \& Bock 1978; Vinod \& Ullah 1981). Results along these lines will be reported separately.

\section{Conclusion}

Conventional methods of ARTS order estimation, viz. FPE, AIC and MDL were 
assessed on the basis of 1000 synthetic models for true orders 3,5 and 7 each. A heuristic order estimation procedure is suggested which may do uniformly better than these though as a compromise by not doing the best possible at individual orders. The procedure suggested uses three opinions from a conventional criteria and three statistics based on them to derive the order estimate. The objectives of showing that

(a) the problem of ARTS order estimation is still an open problem and that there exist better possibilities than the conventional methods,

(b) the distribution of the estimated order can be improved upon, and

(c) heuristics may do better than the available axiological insights,

are regarded as served.

The authors are grateful to Dr H K Gupta of the National Geophysical Research Institute for encouragement.

\section{References}

Akaike H 1969 Power spectrum estimation through autoregressive model fitting. Ann. Inst. Stat. Math. 21: 407-409

Akaike H 1970 Statistical predictor identification. Ann. Inst. Stat. Math. 22: 203-217

Akaike H 1973 Maximum likelihood identification of Gaussian autoregressive moving average models. Biometrika 60: 255-265

Akaike H 1974 A new look at statistical model identification. IEEE. Trans. Autom. Control 19: 716-723

Alexander S T 1986 Adaptive signal processing, theory and applications (New York: Springer Verlag) pp. 34-45, 99-110

Anderson C A 1963 Simplicity in structural geology. In The fabric of geology (ed.) C C Albritton. Jr (Stanford: Freeman, Cooper) pp. 175-183

Barnett V, Lewis T 1978 Outliers in statistical data (Chichester: John Wiley) pp. 25-48

Bernal J D 1969 Science in history, The emergence of science (Harmondsworth: Pelican) 1: 301

Box G E, Jenkins G M 1976 Time series analysis: forecasting and control (San Francisco: Holder Day) p. 575

Broersen M T, Wensink H E 1993 On finite sample theory for autoregressive model order selection. IEEE Trans. Signal Process. 41: 194-204

Constable S C, Parker R L, Constable C G 1987 Occam's inversion, a practical algorithm for generating smooth models for electromagnetic sounding data. Geophysics 52: 289

Diaconis P, Efron B 1983 Computer intensive methods in statistics. Sci. Am. 248: 116

Hoel P G 1966 Introduction to mathematical statistics (New York: John Wiley) p. 44

Judge G G, Bock M E 1978 The statistical implications of pretest and Stein-rule estimators in econometrics (Amsterdam: North Holland) p. 340

Kay S M, Marple Jr S L 1981 Spectrum analysis - a modern perspective. Proc. IEEE 69: $1380-1419$

Makhoul J 1975 Linear prediction: A tutorial review. Proc. IEEE 63: 561-580

Moharir P S 1985 Predictive deconvolution. Parts 1 \& 2. J. Inst. Electron. Telecommun. Eng. 31: $111-121,157-168$

Moharir P S 1988 Central tendency and dispersion: a broader look Proc. 6th Indian Geol. IGC, Roorkee, pp. 217-219

Moharir P S 1991 Non-optimality and positive Occamic gradient. Inst. Eng. India J. 71: 116-121

Moharir P S 1993 Induced Condorcet paradox. In Signal processing and communications (eds) T V Sreenivas, K V S Hari (New Delhi: Tata McGraw-Hill) 129-134 
Proakis J G, Manolakis D G 1989 Introduction to digital signal processing (London: Maxwell Macmillan) 203-205

Rissanen J 1983 A universal prior for the integers and estimation by minimum description length. Ann. Stat. 11: 417: 431

Shibata R 1976 Selection of order of an autoregressive model by Akaike's information criterion. Biometrika 73: 117-126

Vinod H D, Ullah 1981 Recent advances in regression methods (New York: Marcel Dekker) 